\title{
Median and Morphological Specialized Processors for a Real-Time Image Data Processing
}

\author{
Kazimierz Wiatr \\ Institute of Electronics, AGH Technical University of Cracow, Mickiewicza 30, 30-059 Krakow, Poland \\ Email:wiatr@uci.agh.edu.pl
}

Received 29 July 2001 and in revised form 12 October 2001

\begin{abstract}
This paper presents the considerations on selecting a multiprocessor MISD architecture for fast implementation of the vision image processing. Using the author's earlier experience with real-time systems, implementing of specialized hardware processors based on the programmable FPGA systems has been proposed in the pipeline architecture. In particular, the following processors are presented: median filter and morphological processor. The structure of a universal reconfigurable processor developed has been proposed as well. Experimental results are presented as delays on LCA level implementation for median filter, morphological processor, convolution processor, look-up-table processor, logic processor and histogram processor. These times compare with delays in general purpose processor and DSP processor.
\end{abstract}

Keywords and phrases: image processing, median specialized processor, morphological specialized processor, real-time vision system.

\section{TASKS OF REAL-TIME IMAGE ANALYSIS}

The vision signal real-time processing for the needs of control systems require high computation powers. Therefore, methods for fast implementation of the processing algorithms have to be looked for. In the bibliography, many attempts can be found to formulate the algorithms so that their implementation time is as short as possible. However, the author took an effort to search qualitatively different solutions which would be on the one hand related to using specialized hardware structures for implementing various operations and on the other hand to use such intercommunication of those that the architecture is as effective as possible.

Here below, the structure of the vision system is presented and its tasks are singled out. In the algorithms of image analysis, several levels of image processing can be singled out $[1,2]$. Most often, three levels are provided (Figure 1). The lowest level of image analysis (I), called the vision signal preprocessing is aimed at: eliminating the interference, drawing the object out of its background, edge detection, adjusting the object greyness level from the histogram, histogram balancing, and so forth. The middle level of image analysis (II) performs the image segmentation, the object localization, recognizes the image shape and singles out the shape specific features. The highest level (III) is the analysis of the complicated scene: the object movement detection, the object current control, presetting the parameters for low and middle level image processing and analysis.

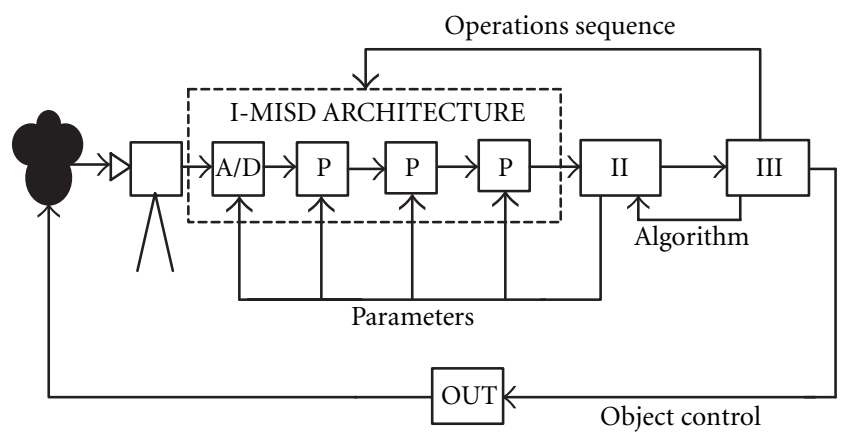

FIGURE 1: A real-time image processing levels.

The vision system structure in Figure 1 shows the feedbacks between various levels of the vision system. The results of the (II) and the (III) image processing stage can affect the parameters and the sequence of operations performed on the (I) lowest level and the processing algorithms on the (II) level.

This feature is of special importance when hardware methods are used for pre-processing. Under such circumstances, substantial flexibility of the system can be achieved by using reconfigurable hardware structures. New opportunities here are given by programmable systems of high integration scale, FPGA type stored in the internal SRAM store, enabling ON-LINE change both of the processing parameters and of the processing algorithms. In his work, the author used the programmable Xilinx FPGA systems, series XC4000 [3]. 


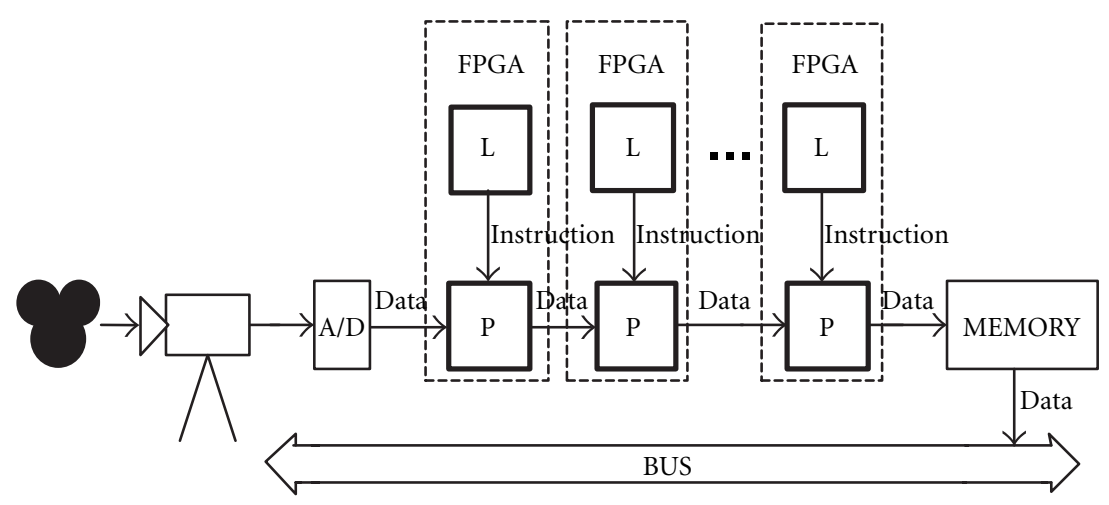

FIGURE 2: MISD architecture of specialized hardware processors.

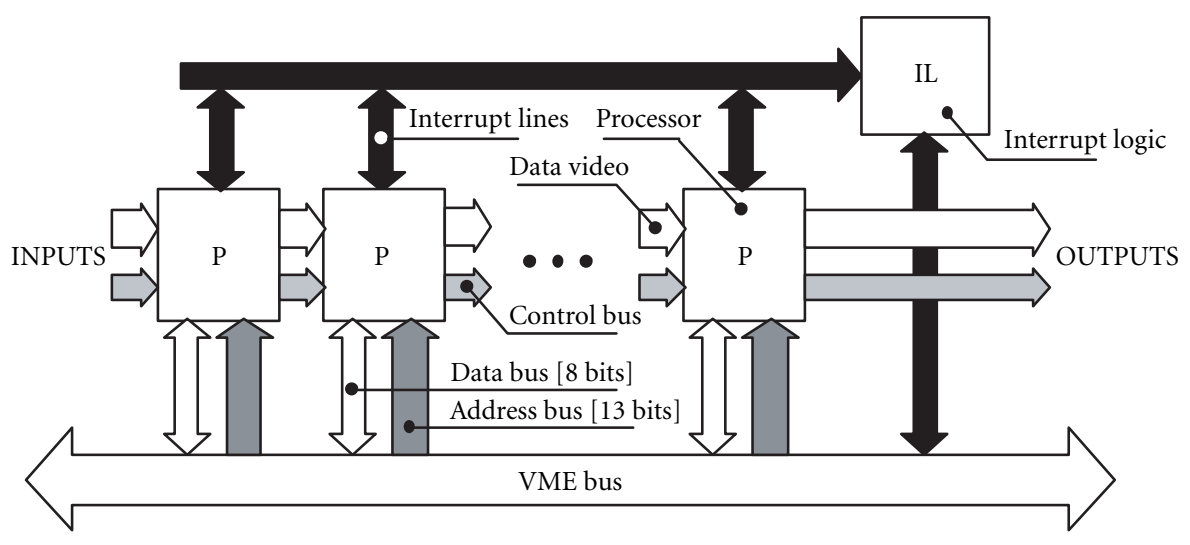

FIGURE 3: A specialized hardware processors architecture.

\section{DEDICATED PIPELINED ARCHITECTURE FOR IMAGE PROCESSING}

The goal of these works was to develop a multiprocessor architecture which-due to the computation elements used and to their interconnection-would result in a very short implementation time of the image pre-processing. In particular, the time has been reduced down to $40 \mathrm{~ms}$ by the provided image acquisition standard of $25 \mathrm{~Hz}$ frequency.

The efficiency of effective use of the multiprocessor structure is related to the optimized assignment of the computation tasks to various processors and to the proper data transfer between them, as well as to their synchronized operation. Such requirements necessitate that not only specialized hardware processors are used in the image processing, but dedicated architectures of multiprocessor systems as well.

A survey of possible architectures of multiprocessor systems has been performed. Considering that the vision data to be processed are great blocks of data (for an image of 512 pixels $\times 512$ pixels the block capacities are $256 \mathrm{kB}$ ), the duration of their transmission between the processors is equally important as the duration of each operation performed by the processors. The most effective here is the multiproces- sor pipelined system based on MISD architecture (Multiple Instruction-stream Single Data-stream) [2, 4] implemented in FPGA structures (Figure 2).

For the purpose of video signal pipelined processing, a bus standard has been developed for the cooperation of specialized processors performing the image pre-processing. In the pipelined mode the video data ( 8 bits) are transferred as well as the control signals securing the synchronized operation of the processors. Such solution of the pipelined bus enables making use of various independently designed processor modules configurated in a system according to what is needed. For each one of them it is possible that its operating position in the pipeline is physically changed, due to which the algorithm of the video signal processing can be flexibly formed and matched with the specific conditions.

Extra opportunities to shape the form produced in the image transformation system are a result of a routine selection of factors (e.g., the convolution matrix) engaged in the hardware processor process, transferred from the external bus (e.g., VME bus) level which is not engaged in the pipelined transfer of the vision data (Figure 3 ).

The pipelined architecture in Figure 3 shows hardware processors $\mathrm{P}$ interconnected by a pipelined bus composed 


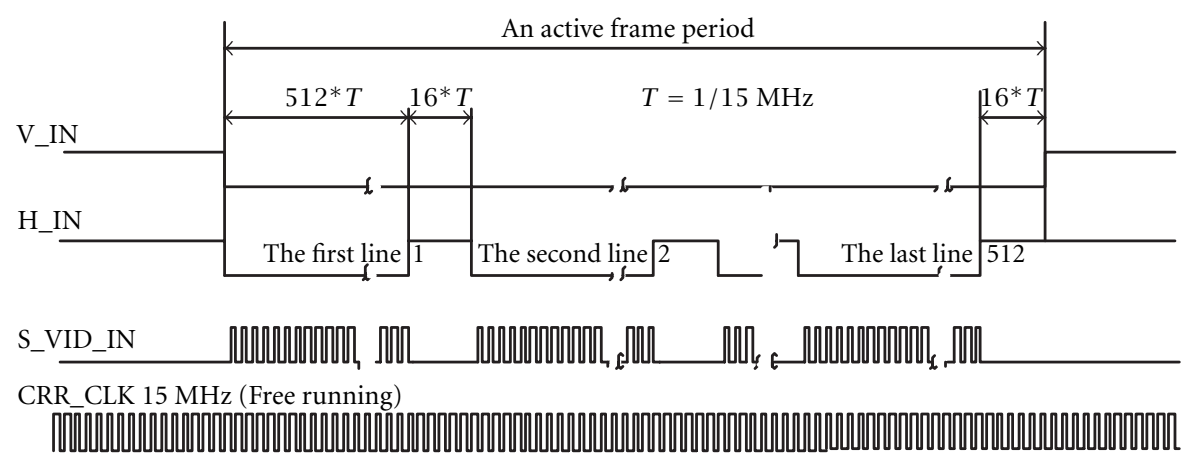

FIGURE 4: Data pipeline format.

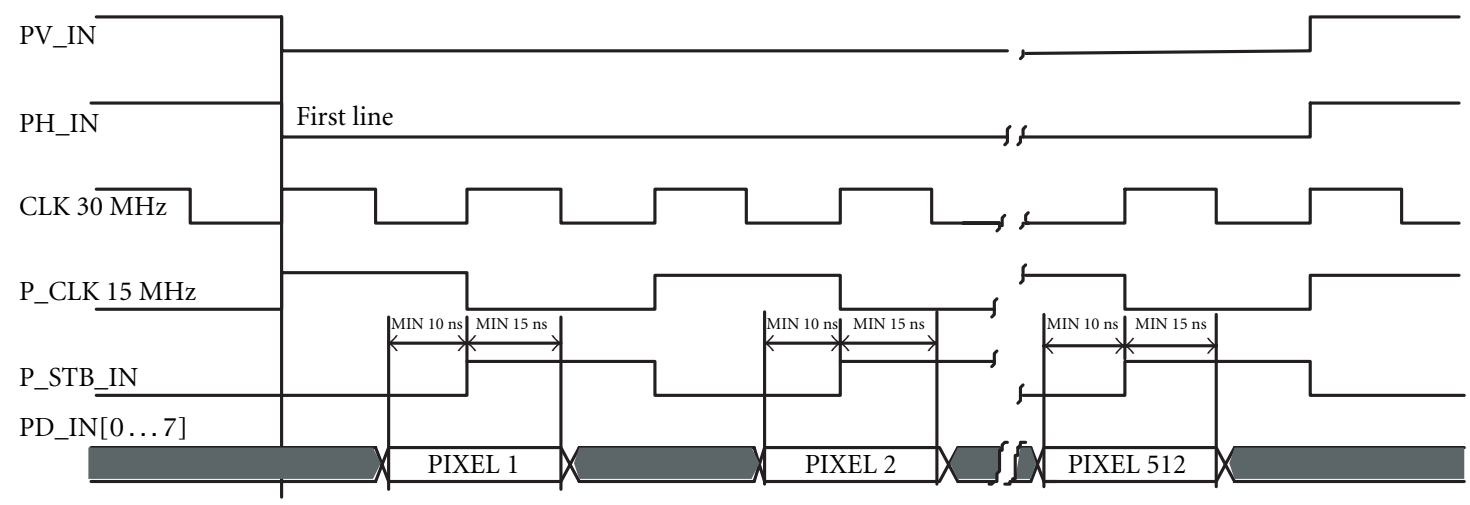

FIgURE 5: Detailed timings of one line.

of the video data and the control signals [5]. The hardware processors are accessible from the VME bus level. The logic module IL serves the interrupt signals and their transfer onto VME bus.

Pipelined processing of a video signal from the camera claims very high marks from the pipelined processor which has to process the pixel completely, before the next portion of information (next pixel) comes. The time available for the pipelined processor is strictly related to the sampling frequency of an A/D converter which is connected to the analogue camera output. This time is resulting from the time of a signal image processing by the camera and its division into lines and pixels.

Usually for the purpose of image analysis, a square (geometrically) field of image is provided, which is divided into square pixels. In order to preserve the square field, the reduced length of a line to be analyzed is $3 / 4 \times 512 / 575 \times 52 \mu \mathrm{s}(52 \mu \mathrm{s}$ standard duration of the visible part of a single line). The above considerations result in the sampling frequency of the analogue/digital (A/D) converter, which value is represented by the equation

$$
f=\frac{K * N_{\mathrm{ver}}}{t_{\mathrm{hor}}}=14,75 \mathrm{MHz},
$$

where $K=N_{\text {hor }} / N_{\text {ver }}=4 / 3$ image proportions; $N_{\text {hor }}=575$ number of the horizontal lines visible on the screen; $t_{\text {hor }}=$ $52 \mu$ s active time of line scanning (PAL).
The video signal is transferred as a series of samples (for the system here described, it has been assumed that 1 sample $=1$ pixel $=8$ bits $)$ in the image frames following each other. Each frame is composed of 512 lines a 512 pixels in each line. The data flow rate through the bus is $15 \mathrm{MB} / \mathrm{s}$. The resolution of a bus for video data processing is 8 bits; thus, an 8-bit input bus of video data (PD_INO..7) and a same output bus (PD_OUTO..7) is connected to each module.

Image synchronization for each processor module is achieved by the input signals (PH_IN, PV_IN) of horizontal and vertical extinction (Figure 4 ) and by the output signals (PH_OUT, PV_OUT), respectively. The output signals are generated by the control logic of each module, and they appear with a delay corresponding to the one resulting from the duration of the video signal processing by the particular processor (Figure 5). The subsequent samples (pixels) are introduced into the module during the growing edge of the strobe signal of video data (P_STB_IN). At the module output, a corresponding output signal (P_STB_OUT) is generated, with the same reservation as for the extinction signals.

\section{MEDIAN SPECIALIZED PROCESSOR}

The median filtration purpose is to compute the median value of the element to be processed and its surroundings. Various dimensions of the port in consideration, which is the element surroundings, are possible. Two types of median filtration 


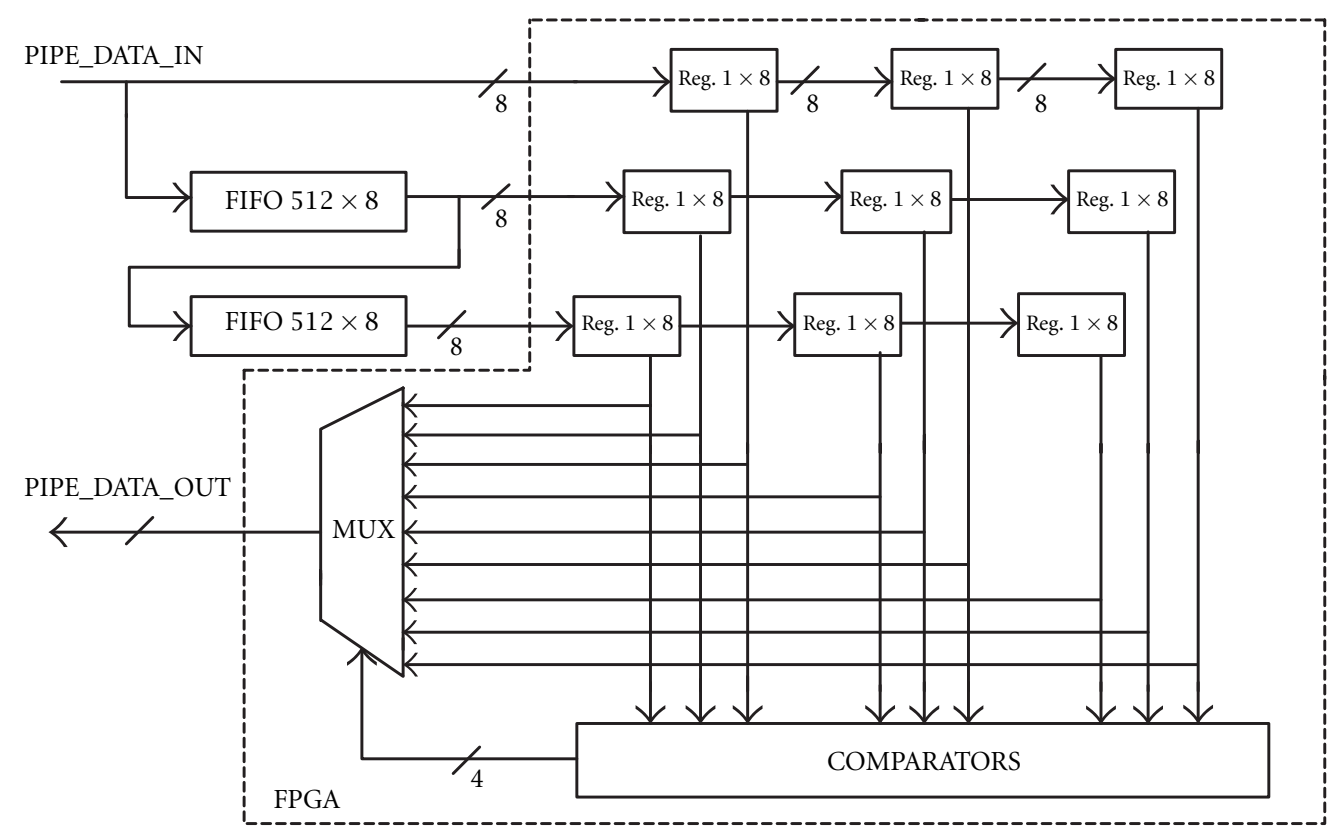

Figure 6: Median processor.

have been assumed to be sufficient: 5-element median and 9-element median $[6,7,8,9]$.

A median filtration processor module (median processor) is to include:

- delay lines, enabling simultaneous access to the necessary element surroundings;

- a group of comparators to compare the element values;

- an output multiplexer and a pipeline bus interface.

Actually, it means the median processor module is to be furnished with two delay lines 512 words long (one line of the image) and 8 bits wide (256 grey levels). In order to secure a simultaneous access to the entire element surroundings for 9 -element median, the processor module is to include 9 registers by 8 bits which outputs are supplied to the group of comparators and to the multiplexer (for a 5-element median, it will be 5 registers by 8 bits, respectively) as per Figure 6 .

The operating speed of a pipeline bus $(15 \mathrm{MHz})$ necessitates a parallel data processing structure to be used. Thus, for example, for a 9-element median 36 pairs of 8-bit numbers are simultaneously compared in the comparator block (for a 5-element median, pairs: 1-2, 1-3, 1-4, 1-5, 2-3, 2-4, $2-5,3-4,3-5,4-5$ are compared by 10 comparators). In the programmable system Xilinx FPGA used for the logic implementation, 8-bit comparators COMP M8 were made use of for this purpose (they are available in XC4000 series only, as library elements, and they use 5 CLB elements). The comparator outputs are the input address for the median value recording/selection memory. The output of this memory is the address for the multiplexer. Each comparator has two outputs GT (greater than), active by $\mathrm{H}$ state for $\mathrm{A}<\mathrm{B}$, and LT (less than), active by $\mathrm{H}$ state for $\mathrm{A}<\mathrm{B}$ (with $\mathrm{A}=\mathrm{B}$, both outputs are inactive).
Thus, for a 9-element median the memory size will be 72 inputs (WE_MEM) and 4 outputs (WY_MEM). For a 5element median, the memory size will be 20 inputs and 3 outputs, respectively. Implementation of the median value recoding/selection memory in FPGA structure enables its realization both as a ROM and RAM memory. Implementation as RAM memory is somewhat slower and more resources are being used; however, it enables the new pixel value selected (not necessarily the median one) to be dynamically shaped by ON-LINE writing the new contents of RAM memory which is responsible for recording. The delay introduced for 5-element median is $68 \mu \mathrm{s}$ (two image lines).

For the purpose of hardware implementation of the median processor, FIFO buffers (First In-First Out) IDT 72210 produced by IDT (Integrated Device Technology) have been used. The buffer organization is $512 \times 8$ bits and the access time is $12 \mathrm{~ns}$. For logic implementation, programmable Xilinx FPGA system has been used, its designation being XC4005-5 PC84 [3].

\section{MORPHOLOGICAL SPECIALIZED PROCESSOR}

As an example this paper presents morphological pipeline processor. Morphological operations include a wide class of transformations realized in binary images $[10,11,12,13]$. These are operations from the border line of (I) and (II) levels of image transformations. One can implement in the elaborated pipeline architecture-a morphological processor, whose application is limited to simple morphological operations (e.g., erosion or dilatation).

Figure 7 shows a diagram of a morphological processor, meant to work in the pipeline architecture. The whole logic is placed in an FPGA programmable structure. Since a mor- 


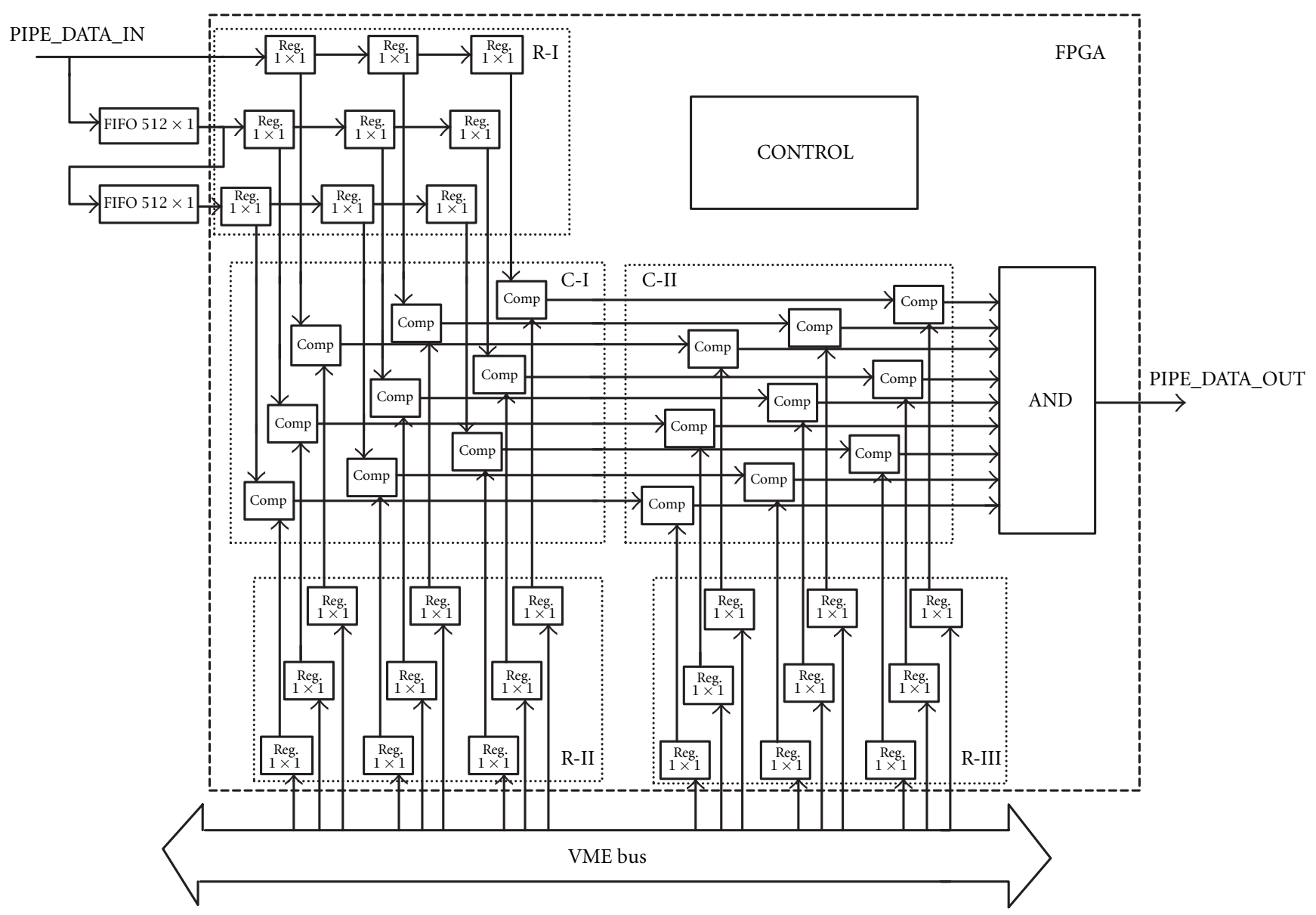

FIgURE 7: Morphological processor.

phological processor realizes context operations (the result of operation depends on the quality of the transformed point environment), it was necessary to use two external delay lines of $512 \times 1$ bit organization (morphological operations in binary image).

The processor logic consists of three register groups ( 9 1-bit registers in each group) and two comparator groups (9 1-bit comparators in each group). The first register group (RI) is meant to memorize the transformed point together with its environment. Information stored in these registers comes from the pipeline architecture and two delay lines, thanks to which on FPGA chip input, three successive image lines appear. The second register group (R-II) includes values of individual points of the structural element, but what is important on this level are only 0 and 1 values. The third register group (R-III) memorizes, which points of the structural element are not taken into account in the course of comparing (value $x$ ).

Thanks to the simultaneous access to these values, one can, within the first set of comparators (C-I), compare values of image points and the structural element, and next in the second set of comparators (C-II), pass the results of these comparisons, which refer to the points disregarded (value $x$ ).

Next, in block AND, logical product of the second com- parator set outputs is performed (the comparator output is set to 1 for accordant values of inputs). The logical product value is fed on the morphological processor output. In Figure 7 the logic controlling the operation of the processor is passed (synchronization with pipeline architecture, entering data in individual registers, etc.).

To elaborate a morphological processor, buffer FIFO (IDT72210) have been used as delay lines, made by IDT (Integrated Device Technology). They are FIFO buffer of surplus capacity (organized as 512 8-bit words, instead of 512 1-bit words), but thanks to this, one could keep some homogeneous of the components applied in the construction of individual processors. To realize the remaining logic, a programmable chip FPGA, made by Xilinx, was used, marked XC4002-5PC84C [3].

\section{RECONFIGURABLE UNIVERSAL PIPELINED PROCESSOR}

The exemplary hardware processors herein described to operate in the pipelined bus developed by the author are not open to the changes in the image processing algorithm. Particularly, it is impossible to change the operation sequence, which would necessitate changes of the given module posi- 


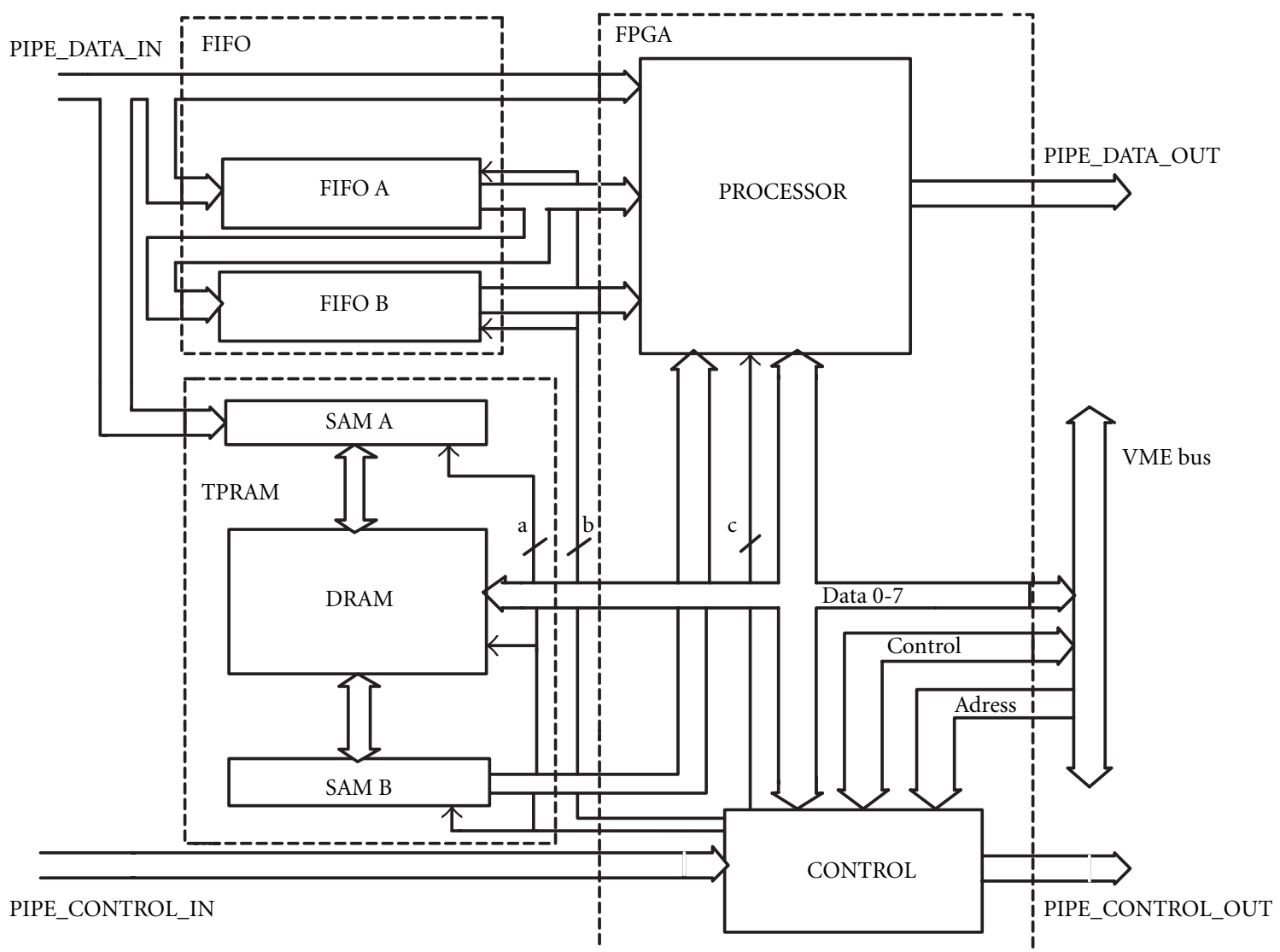

FIGURE 8: Reconfigurable pipelined processor.

tions in the pipeline architecture slots. New options in this area are related to the use of FPGA programmable systems of high integration scale, their configuration to be written in RAM memory $[14,15,16]$.

The author's universal reconfigurable pipelined processor is a module comprising three parts: FPGA structure, tripleport memory (TPRAM), two FIFO buffers.

Such uniform hardware structure developed enables implementation of any processor described earlier, in some implementations certain hardware resources remaining inactive. This enables any sequence of pre-processing operations of the images produced by hardware processors, with no need to physically relocate the dedicated modules of specialized processors. Changes can be made during the system normal operation. Usually, a need for such change is a result of changes on the scene observed (changes of weather, of the day-time, of the followed object, etc.).

Figure 8 shows the diagram of the author's reconfigurable pipelined processor. It has been based on Xilinx FPGA programmable system involving substantial resources of internal logic and a great number of input/output system (optionally, a system XC4005-PQ160 through XC4010-PQ160) [3]. Two FIFO buffers (IDT72210) of $512 \times 8$ bits enable a simultaneous processor access to the entire surroundings of the pixel to be processed (surroundings $3 \times 3$ ). The triple-port memory TPRAM (MT43C4257) enables the logic operations to be performed on two images, one from the camera and the other (written by the master processor) from the bus, or it is one of the preceding images.

\section{CONCLUSION}

The above structure is very much competitive in view of the finite capabilities of conventional microprocessors to enhance their computation power and of the operating frequency of their clocks. With this structure, the cycles of instruction and data reception are eliminated, and the operations themselves are performed in parallel.

The performance time of several exemplary operations of image pre-processing is as follows [17]:

- median filtering $68 \mu \mathrm{s}$,

- morphological processor $68 \mu \mathrm{s}$,

- $3 \times 3$ convolution processor $102 \mu \mathrm{s}$,

- look-up-table 66 ns,

- subtraction of two frames $132 \mathrm{~ns}$,

- histogram processor $66 \mathrm{~ns}$.

The modern Texas signal processor TMS320C80 perform such pre-processing operations within the following time [18]:

- median filtering $10.7 \mathrm{~ms}$, 
- morphology binary erosion $12.7 \mathrm{~ms}$,

- morphology binary closing $21.2 \mathrm{~ms}$,

- $3 \times 3$ convolution $19.4 \mathrm{~ms}$,

- histogram $4.3 \mathrm{~ms}$,

- logic operation $5.4 \mathrm{~ms}$.

The dedicated module MAMBA with modern microprocessor Pentium II - $466 \mathrm{MHz}$ perform such preprocessing operations within the following time [19]:

- $3 \times 3$ convolution $6.8 \mathrm{~ms}$,

- histogram $4.0 \mathrm{~ms}$,

- look-up-table $3.0 \mathrm{~ms}$,

- add or subtract two images $2.7 \mathrm{~ms}$.

The pipelined bus module for testing was placed in a cassette with VME bus. The works were supervised by a real-time operation system OS-9 installed on FORCE SYS68K/CPU32 module (Motorola MC68030 microprocessor) together with SYSTEM-PAK I/MGR graphic package operating in conjunction with EKF SAGA 6/7842 graphic controller.

\section{ACKNOWLEDGEMENT}

This work was supported by the Polish Science Committee.

\section{REFERENCES}

[1] P. Chalermwat, N. Alexandridis, P. Piamsa-Nga, and M. O'Connell, "Parallel image processing in heterogeneous computing network systems," in Proceedings of the IEEE International Conference on Image Processing (ICIP96), Lausanne, Switzerland, September 1996, pp. 16-19.

[2] C. L. Wang, P. B. Bhat, and V. K. Prasanna, "High-performance computing for vision," in Proceedings of the IEEE, vol. 84, pp. 931-946, July 1996.

[3] XILINX, "The programmable logic data book," Xilinx Inc., 1999, San Jose, CA.

[4] R. Duncan, "A survey of parallel computer architectures," Computer, vol. 23, no. 2, pp. 5-16, 1990.

[5] A. Antola, A. Avai, and L. Breveglieri, "Modular design methodologies for image processing architectures," IEEE Trans. on VLSI Systems, vol. 1, no. 4, pp. 408-414, 1993.

[6] A. Kundu and J. Zhou, "Combination median filter," IEEE Trans. Image Processing, vol. 1, no. 3, pp. 422-429, 1992.

[7] G. Qiu, "An improved recursive median filtering scheme for image processing," IEEE Trans. Image Processing, vol. 5, no. 4, pp. 646-648, 1996.

[8] X. Yang and P. S. Toh, "Adaptive fuzzy multilevel median filter," IEEE Transactions on Image Processing, vol. 4, no. 5, pp. 680682, 1995.

[9] B. Zeng, "Convergence properties of median and weighted median filters," IEEE Trans. on Signal Processing, vol. SP-42, no. 12, pp. 3515-3518, 1994.

[10] F. Cheng and A. N. Venetsanopoulos, "An adaptive morphological filter for image processing," IEEE Trans. Image Processing, vol. 1, no. 4, pp. 533-539, 1992.

[11] H. J. A. M. Heijmans, "Composing morphological filters," IEEE Trans. Image Processing, vol. 6, no. 5, pp. 713-723, 1997.

[12] X. C. Jin, S. H. Ong, and Jayasooriah, "A domain operator for binary morphological processing," IEEE Trans. Image Processing, vol. 4, no. 7, pp. 1042-1046, 1995.

[13] P. Maragos, "Differential morphology and image processing," IEEE Trans. Image Processing, vol. 5, no. 6, pp. 922-937, 1996.
[14] P. J. Bakkes, J. J. du Plessis, and B. L. Hutchings, "Mixing fixed and reconfigurable logic for array processing," in Proceedings of IEEE Workshop on FPGAs for Custom Computing Machines, Napa, CA, April 1996, pp. 118-125.

[15] W. Luk, N. Shirazi, and P. Y. K. Cheung, "Modeling and optimizing run-time reconfigurable systems," in Proceedings of the IEEE Symposium on FPGAs for Custom Computing Machines, California, November 1996, pp. 167-177.

[16] J. Villasenor, C. Jones, and B. Schoner, "Video communications using rapidly reconfigurable hardware," IEEE Transactions on Circuits and Systems for Video Technology, vol. 5, no. 6, pp. 565-567, 1995.

[17] K. Wiatr, "Specialized architecture of dedicated hardware processors for a real-time image data pre-processing," in Proceedings of the EUROMICRO International Conference: Real-Time Systems, Toledo, 1997.

[18] UWICL, "UWICL 3.0 function list and performance figures," University of Washington Image Computing Library, http://icsl.ee.washington.edu/projects/iclib/.

[19] CORECO, "Mamba benchmarks," Coreco Inc., 2000, StLaurent, Quebec.

Kazimierz Wiatr received the M.Sc. and Ph.D. degrees in electrical engineering from the AGH Technical University of Cracow in 1980 and 1987, respectively. His Ph.D. thesis dealt with the microprocessor systems for the real-time applications. From June 1991 to December 2000, he was awarded four research grants from Polish Committee of Science Research. During this time, he worked

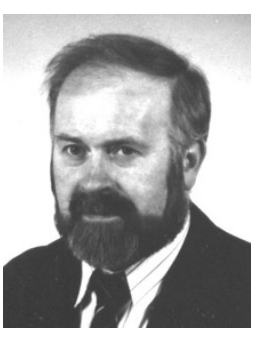
on the implementation of high-speed digital signal-processors with an emphasis on real-time image pre-processing used FPGA structures. These works resulted in over 70 technical papers, monograph title Pipeline Architecture of Specialised Hardware Processors for Real-Time Image Pre-Processing and two rewards from the Polish Ministry of Education. He received in 1999 the D.Sc. degree from the University of Technology in Lodz and in 2001 Professor AGH position. He is a reviewer of IEE Proceedings and IEEE Transactions. 